PROCEEDINGS OF THE

AMERICAN MATHEMATICAL SOCIETY

Volume 68, Number 1, January 1978

\title{
MAXIMAL SUBALGEBRAS OF CENTRAL SEPARABLE ALGEBRAS
}

\author{
M. L. RACINE ${ }^{1}$
}

\begin{abstract}
Let $\boldsymbol{A}$ be a central separable algebra over a commutative ring $R$. A proper $R$-subalgebra of $A$ is said to be maximal if it is maximal with respect to inclusion.

Theorem. Any proper subalgebra of $A$ is contained in a maximal one. Any maximal subalgebra $B$ of $A$ contains a maximal ideal $m A$ of $A, m$ a maximal ideal of $R$, and $B / \mathrm{m} A$ is a maximal subalgebra of the central simple $R / \mathrm{m}$ algebra $A / \mathrm{m} A$.

More intrinsic characterizations are obtained when $R$ is a Dedekind domain.
\end{abstract}

In [3] and [4] Dynkin studied maximal subalgebras of simple Lie algebras over an algebraically closed field of characteristic zero and the connection between these and the maximal subgroups of the classical groups. In [7] and [8] the maximal subalgebras of the following classes of central simple algebras were determined: associative, associative with involution, alternative and Jordan. In this paper maximal subalgebras of central separable algebras over a commutative ring $R$ are considered. The ideal structure of such algebras is entirely determined by that of $R$ so it is interesting to consider one-sided ideals and to a lesser extent subalgebras.

We recall some results of [7]. Let $F$ be a field and $A$ a (finite dimensional) central simple algebra over $F$. So $A \cong M_{n}(D), D$ a finite dimensional division algebra over its center $F$ and $A$ acts on an $n$-dimensional left $D$ vector space $V$. By a subalgebra of $A$ we understand an $F$ subspace of $A$ which is closed under multiplication, and by maximal subalgebra, a proper subalgebra which is maximal with respect to inclusion. If $A=F$ then 0 is the unique maximal subalgebra of $A$. If $A \neq F$ then all maximal subalgebras of $A$ contain 1 and they are exactly the subalgebras of the form:

(I) $S(W)=\{a \in A \mid W a \subset W\}, W$ any nonzero proper subspace of $V$.

(II) $C_{A}(E)$ the centralizer of $E$ a field extension of $F$ lying in $A$, without intermediate subfields (i.e. $F \subsetneq L \subset E, L$ a field $\Rightarrow L=E$ ).

Subalgebras of type I can be described intrinsically. If $e \in A$ is a projection of $V$ onto $W$ and $f=1-e$ then

Received by the editors July 27, 1976 and, in revised form, February 14, 1977.

AMS (MOS) subject classifications (1970). Primary 16A16, 13F05.

Key words and phrases. Central separable algebra, maximal subalgebra, Dedekind domain.

'This research was supported in part by the National Research Council of Canada (NRC Grant No. A9109). 


$$
S(W)=f A \boxplus e A e=f A f \oplus f A e \oplus e A e .
$$

While $W=V \operatorname{Rad}(S(W))$ is uniquely determined, $e$, of course, is not.

Our aim is to extend these results to central separable algebras over a commutative ring $R$. All facts concerning these for which no precise reference is given can be found in [2]. We will need the following

LEMMA. Let $N$ be a proper submodule of a finitely generated module $M$ over a commutative ring $R$. Then there exists a maximal ideal $m$ of $R$ such that $N+\mathfrak{m} M$ is a proper submodule of $M$.

Proof. If not, then $N+\mathrm{m} M=M \forall \mathrm{m} \in \operatorname{Max}(R)$. The $R$ module $M / N$ is still finitely generated and since $M=N+\mathfrak{m} M$ then $\mathrm{m}(M / N)=M / N$ $\forall \mathfrak{m} \in \operatorname{Max}(R)$. Therefore $M / N=0([2,1.8])$ a contradiction.

TheOREM. Let $A$ be a central separable $R$ algebra. Then any proper subalgebra of $A$ is contained in a maximal one. Any maximal subalgebra $B$ of $A$ contains a maximal ideal $\mathrm{m} A$ of $A, \mathrm{~m}$ a maximal ideal of $R$, and $B / \mathrm{m} A$ is a maximal subalgebra of the central simple $R / \mathrm{m}$ algebra $A / \mathrm{m} A$.

Proof. If $B$ is a proper subalgebra of $A$, since $A$ is finitely generated as an $R$ module, the Lemma implies that $B+\mathrm{m} A$ is a proper submodule of $A$ for some $\mathfrak{m} \in \operatorname{Max}(R) . B+\mathfrak{m} A$ is a subalgebra and $B+\mathfrak{m} A / \mathfrak{m} A$ is a proper subalgebra of $A / \mathrm{m} A$ and hence contained in a maximal one, say $C^{\prime}$. Let $C$ be the inverse image of $C^{\prime}$ in $A$. Then $B \subset C$ which is a maximal subalgebra of $A$. If $B$ were maximal to start with, then $\mathrm{m} A \subset B$ since $B+\mathrm{m} A$ is a proper subalgebra, and $B / \mathrm{m} A$ is a maximal $R / \mathrm{m}$ subalgebra of $A / \mathrm{m} A$.

Given a maximal subalgebra $B$ of a central separable $R$-algebra $A$ then $\mathfrak{m}=\{\alpha \in R \mid \alpha A \subset B\}$ is uniquely determined. We say that $B$ is of type $\mathrm{I}$, of type II, or trivial according as $\bar{B}=B / \mathrm{m} A$ is maximal of type I or II, or 0 as a subalgebra of the central simple $R / \mathrm{m}$ algebra $\bar{A}=A / \mathrm{m} A$. The type of $B$ is well-defined by the uniqueness of $m$ and that of the type of $B / \mathrm{m} A$. It would be nice to give an intrinsic description of $B$, that is, without passing to $A / \mathrm{m} A$.

Proposition 1. If $B$ is a trivial maximal subalgebra of a central separable $R$ algebra $A$ then either $A=R$ and $B=\mathfrak{m}$ a maximal ideal of $R$, or $R=S \oplus T$, where $S$ and $T$ are subrings of $R, A=S \oplus A_{0}, A_{0}$ a central separable $T$ algebra, and $B=\mathfrak{m} A$ where $\mathfrak{m}=\mathfrak{m}^{\prime}+T, \mathfrak{m}^{\prime}$ a maximal ideal of $S$. Conversely if $A$ is as above then for any $\mathrm{m}^{\prime} \in \operatorname{Max}(S)$ the subalgebra $\mathrm{m}^{\prime}+A_{0}$ is maximal and trivial.

Proof. Let $B$ be a trivial maximal subalgebra of a central separable $R$ algebra $A$. Then $B=\mathrm{m} A$ for some $\mathrm{m} \in \operatorname{Max}(R)$ and $A / \mathrm{m} A$ is a field. Therefore $A / \mathrm{m} A=R / \mathrm{m}$. Now $R$ is an $R$ module direct summand of $A$. If $A=R$ then we are in the first case above. If not then $A=R \oplus M$ as an $R$ module. Therefore $\mathfrak{m} A=\mathfrak{m} \oplus \mathfrak{m} M=\mathfrak{m}+M$ and $\mathfrak{m} M=M$. Since $A$ is finitely generated projective so is $M$. Let $m_{1}, \ldots, m_{k} \in M$, $f_{1}, \ldots, f_{k} \operatorname{Hom}_{R}(M, R)$ be a dual basis for $M$. Since $m_{i} \in m M$ we have 
$m_{i}=\sum_{j=1}^{k} \alpha_{i j} m_{j}, \alpha_{i j} \in \mathfrak{m}$ and the trace ideal of $M, \tau M \subset \mathfrak{m}$. But $R=$ Ann $M$ $\oplus \tau M$, Ann $M$ the annihilator of $M$. Let $\pi: R \oplus M \rightarrow R$ be the canonical projection and $g_{i}: M \rightarrow R$ be defined by $g_{i}(m)=\pi\left(m m_{i}\right), 1 \leqslant i \leqslant k$. Then $g_{i} \in \operatorname{Hom}_{R}(M, R)$ and $g_{i}(M) \subset \tau M$. Thus $A_{0}=\tau M+M$ is a subalgebra of $A$. Let $S=$ Ann $M, T=\tau M$. Since $A$ is $R$ central, $A_{0}$ must be $T$ central. Taking the component of a separability idempotent of $A$ coming from $A_{0} \otimes_{T} A_{0}^{o}$, where $A_{0}^{o}$ denotes the opposite algebra of $A_{0}$, we obtain a separability idempotent for $A_{0}$ which is therefore a central separable $T$ algebra. The maximality of $\mathfrak{m}$ implies the maximality of $\mathfrak{m}^{\prime}=\mathfrak{m} \cap S$ and we have the first half of the proposition. The converse is clear.

Let us consider next subalgebras of type I. If $e \neq 0,1$ is an idempotent of $A$, let $f=1-e$. Then $B=e A e+f A+\mathfrak{m} A$ is a maximal subalgebra of $A$ of type $I$ for any $\mathfrak{m} \in \operatorname{Max}(R)$. The converse, namely, if $B$ is a maximal subalgebra of $A$ of type I containing $\mathrm{m} A$ then $B=e A e+f A+\mathfrak{m} A$ for some idempotent $e \in A$, is not true in general as the following example will show. Let $R=\mathbf{Z}_{(p)}$ the integers localized at $p$ an odd prime and let $1, i, j, k$ be the standard basis for the Hamiltonian quaternions. The algebra $A=R 1+R i$ $+R j+R k$ is a central separable $R$-algebra (see [6]) which contains no nontrivial idempotent. However $A /(p) A \cong M_{2}(\mathrm{Z} / p \mathrm{Z})$ the $2 \times 2$ matrices with entries in the field with $p$ elements. Therefore $A$ has maximal subalgebras of type $\mathrm{I}$, but none of the form $e A e+f A+(p) A$. Some positive results are given in

Proposition 2. If (1) $R$ is Henselian, $A$ any central separable $R$-algebra, or (2) $R$ is a Dedekind domain and $A=\operatorname{End}_{R} M, M$ an $R$ progenerator, then any maximal subalgebra of $A$ of type $I$ has the form eAe $+f A+\mathfrak{m} A$ for a suitably chosen idempotent $e \in A, f=1-e$, and a maximal ideal $m$ of $R$.

Proof. (1) follows immediately from Azumaya's result [1] that idempotents of $A / \mathrm{m} A$ lift to idempotents of $A$.

In case (2) while not every idempotent of $A / \mathrm{m} A$ lifts to an idempotent of $A$ we will show that given a subspace $W$ of the $R / \mathrm{m}$ vector space $V=$ $M / \mathfrak{m} M$ then there is always a projection of $V$ onto $W$ that does lift. Since $M$ is a progenerator, $M$ is a faithful finitely generated projective $R$ module. If $B$ is a maximal subalgebra of $A$ of type I containing $\mathrm{m} A$ then $\bar{B}=B / \mathrm{m} A=\{a$ $\in \bar{A} \mid W a \subset W\}$ where $\bar{A}=A / \mathrm{m} A$ and $W \neq 0$ is a proper subspace of $V=M / \mathfrak{m} M\left(\bar{A}=\operatorname{End}_{R / \mathfrak{m}} V\right)$. Choose a set of preimages in $M$ of some $R / \mathfrak{m}$ basis of $W$ and let $N$ be the $R$ submodule of $M$ they generate. So $W=N+\mathfrak{m} M / \mathfrak{m} M$. By Theorem 81.11 of [5] one can choose $x_{1}, \ldots, x_{n} \in$ $M$ such that $M=a_{1} x_{1} \oplus \cdots \oplus a_{n} x_{n}, N=a_{1} b_{1} x_{1} \oplus \cdots \oplus a_{n} b_{n} x_{n}$ where $a_{i}$ are fractional ideals of $R$ and $\mathfrak{b}_{1} \supset \mathfrak{b}_{2} \supset \cdots \supset \mathfrak{b}_{n}$ are uniquely determined ideals of $R$. Let $k$ be the first index such that $\mathfrak{b}_{k} \subset \mathfrak{m}$ and let $P=a_{1} x_{1}$ $\oplus \cdots \oplus \mathfrak{a}_{k-1} x_{k-1}$. Since $\mathfrak{b}_{i} \not \subset \mathfrak{m}$ for $i<k, \mathfrak{b}_{i}+\mathfrak{m}=R$ and $\mathfrak{a}_{i} \mathfrak{b}_{i}+\mathfrak{a}_{i} \mathfrak{m}=$ $\mathrm{a}_{i}$. Therefore $P+\mathrm{m} M=N+\mathrm{m} M$ and $P+\mathrm{m} M / \mathrm{m} M=W$. Now $P$ is a direct summand of $M$, so let $e$ be a projection of $M$ onto $P$ and $f=1-e$. 
Then $\overline{e A e+f A+\mathfrak{m} A}=\bar{B}$ and therefore $B=e A e+f A+\mathrm{m} A$.

In the case where $R$ is arbitrary and $A=\operatorname{End}_{R} M, M$ an $R$ progenerator, then arguing as above one sees that if $B$ is a maximal subalgebra of type $I$ then

$$
B=\{a \in A \mid(N+\mathfrak{m} M) a \subset N+\mathfrak{m} M\}=S(N+\mathfrak{m} M) .
$$

Finally let us consider maximal subalgebras of type II. Let $A$ be a central separable algebra over a Dedekind domain $R, K$ the quotient field of $R$. Then $\Sigma=K \otimes_{R} A$ is central simple algebra over $K$ of dimension say, $n^{2}$ and $A$ is a maximal order of $\Sigma$. If $a \in A$ then its minimal polynomial, $\mu_{a}(x)$, as an element of $\Sigma$ belongs to $R[x]$, [9, Theorem IV 1.4']. Let $m \in \operatorname{Max}(R)$. If $\bar{\mu}_{a}$, the image of $\mu_{a}$ in $R / \mathrm{m}[x]$ under the canonical homomorphism, is irreducible in $R / \mathfrak{m}[x]$ then so is $\mu_{a}$ in $K[x]$. In this case $K[a]$ and $R / \mathfrak{m}[\bar{a}]$ are fields $(\bar{a} \in A / \mathfrak{m} \bar{A})$. If there are no intermediate subfields between $R / \mathfrak{m}$ and $R / \mathrm{m}[\bar{a}]$ then we claim that $K[a]$ is a field extension of $K$ without intermediate subfields. Since $R / \mathrm{m} \cong R_{\mathrm{m}} / \mathrm{m} R_{\mathrm{m}}$, where $R_{\mathrm{m}}$ denotes the localisation of $R$ at $\mathrm{m}$, it suffices to prove the claim for principal ideal domains so assume $\mathrm{m}=(\pi), \pi \in R$. If $K \subsetneq L \subsetneq K[a]$, we may assume $L=K[b]$ and after clearing denominators and subtracting an element of $K$ we may take $b=\alpha_{1} a+\cdots+\alpha_{r} a^{r}, \alpha_{i} \in R, r<\operatorname{deg} \mu_{a}$. Dividing by an appropriate power of $\pi$ we may assume that at least one $\alpha_{i}$ is a unit of $R$. Therefore the image of $b$ in $R / \mathrm{m}[\bar{a}]$ does not belong to $R / \mathrm{m}$. So the extension of $R / \mathrm{m}$ it generates is $R / \mathrm{m}[\bar{a}]$ contradicting the fact that $\operatorname{deg} \mu_{b}<\operatorname{deg} \mu_{a}$. Thus there are no intermediate subfields between $K$ and $K[a]$. Since $[K[a]: K]=$ $[R / \mathrm{m}[\bar{a}]: R / \mathrm{m}]$ and $[\Sigma: K]=[\bar{A}: R / \mathrm{m}]=n^{2}$, the classical double centralizer theorem (e.g. [7, Theorem $\left.4^{\prime}\right]$ ) implies $[C(a): K]=\left[C_{\bar{A}}(\bar{a}): R / \mathrm{m}\right]$. Thus if $B$ is the inverse image in $A$ of $C_{\bar{A}}(\bar{a}) \subset \bar{A}$, then $B=C_{A}(a)+\mathrm{m} A$ and we have proved the first half of

Proposition 3. Let $A$ be a central separable algebra over a Dedekind domain $R$. If $a \in A$ is such that $\bar{\mu}_{a}(x)$ is irreducible in $R / \mathfrak{m}[x]$ for some $\mathfrak{m} \in \operatorname{Max}(R)$ and if the extension $R / \mathrm{m}[\bar{a}]$ of $R / \mathrm{m}$ has no intermediate subfields then $C_{A}(a)+\mathfrak{m} A$ is a maximal subalgebra of $A$ of type II. Conversely any maximal subalgebra of $A$ of type II is of that form.

Proof. We consider first the case when $A=\operatorname{End}_{R}(M), M$ an $R$ progenerator. If $B$ is a maximal subalgebra of $A$ of type II then for some $\mathrm{m} \in \operatorname{Max}(R)$ there is a $b \in B$ such that $\bar{B}=C_{\bar{A}}(\bar{b})$, where $\bar{A}=A / \mathrm{m} A$ and $R / \mathrm{m}[\bar{b}]$ is a field extension of $R / \mathrm{m}$ without intermediate subfields. The minimal polynomial of $\bar{b}, \mu_{b}(x)$, is irreducible in $R / \mathfrak{m}[x]$ and, by the discussion preceding the proposition, it suffices to show that one can choose an $a \in B$ such that $\bar{a}=\bar{b}$ and $\bar{\mu}_{a}=\mu_{\bar{b}}$. Now $\bar{A} \cong M_{n}(R / \mathfrak{m})$ and if $R / \mathfrak{m}[\bar{b}]$ is of degree $k$ then the regular representation provides an embedding $\rho: R / \mathrm{m}[\bar{b}]$ $\rightarrow M_{k}(R / \mathrm{m})$. Since $k \mid n$, say $n=k q$, this provides us with an embedding of $R / \mathrm{m}[\bar{b}]$ in $M_{n}(R / \mathrm{m})$ such that $\bar{b}$ corresponds to $q$ blocks $\rho(\bar{b})$ along the diagonal. By the Skolem-Noether Theorem we may write $M / \mathrm{m} M=V$ as 
$V_{1} \oplus \cdots \oplus V_{q}$, where $V_{i}$ 's are subspaces of dimension $k$ such that $V_{i} \bar{b} \subset V_{i}$. Arguing as in the proof of Proposition 2 we may find submodules $M_{i}$, $1<i<q$ of $M$ such that $M=\bigoplus_{i=1}^{q} M_{i}$ and $M_{i}+\mathrm{m} M / \mathfrak{m} M=V_{i}$. Let $e_{i} \in A$ be the corresponding projections. Thus $1=\sum_{i=1}^{q} e_{i}$ and the $e_{i}$ are mutually orthogonal. Let $b \in B$ be a preimage of $\bar{b}$ and let $a=\sum_{i=1}^{q} e_{i} b e_{i}$. Since $\bar{b} \in \bigoplus_{i=1}^{q} \bar{e}_{i} A e_{i}, \bar{a}=\bar{b}$. Moreover $\mu_{a}(x)$ has degree $\leqslant k$. But $\bar{\mu}_{a}(\bar{b})=0$. Therefore the degree of $\mu_{a}=k$ and $\bar{\mu}_{a}=\mu_{\bar{b}}$.

Let $A$ now be any central separable algebra, $B$ a maximal subalgebra of $A$ of type II and $\mathrm{m}$ the maximal ideal of $R$ mapping $A$ into $B$. Consider $A^{e}=A \otimes_{R} A^{o}$, where $A^{o}$ denotes the opposite algebra of $A ; \rho: A \otimes_{R} A^{o} \rightarrow$ $\operatorname{End}_{R}(A)$ induced by

$$
\rho\left(a_{1} \otimes a_{2}\right)(b)=a_{1} b a_{2}
$$

is an isomorphism and $A^{e}$ is central separable since $A$ is an $R$ progenerator. If $\bar{B}=C_{\bar{A}}(\bar{b})$ with $R / \mathrm{m}[\bar{b}]$ a field extension of $R / \mathfrak{m}$ without intermediate subfields then identify $\bar{b}$ with

$$
\bar{b} \otimes 1 \in A^{e} / \mathrm{m} A^{e} \cong \bar{A} \otimes_{R} A^{o} \cong \bar{A} \otimes_{R / m} \bar{A}^{o} .
$$

By the previous case one can find $c \in A^{e}$ with $\bar{c}=\bar{b} \otimes 1$ and $\operatorname{deg} \bar{\mu}_{c}=$ $\operatorname{deg} \mu_{\bar{b}}$. Let $\nu: A^{e} \rightarrow A$ be induced by $\nu(d)=\rho(d)(1)$. Since

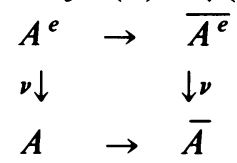

commutes, if we let $a=\nu(c) \in A$ then $\bar{a}=\bar{b}$ and $a$ satisfies $\mu_{c}(x)$ which is therefore equal to $\mu_{a}(x)$.

We end with an example. Let $n \in \mathbf{Z}, n>1$ and $A=M_{n}(\mathbf{Z})$. By Propositions 2 and 3 the maximal subalgebras of $A$ are exactly

(I) $B=e A e+f A e+f A f+p A$, where $e \in A, e^{2}=e \neq 0,1, f=1-e$ and $p$ is any prime of $\mathbf{Z}$.

(II) $B=C_{A}(a)+p A$, where $p$ is any prime of $\mathbf{Z}, a \in A$ whose minimal polynomial $\mu_{a}$ is of degree $q$ a prime divisor of $n$ and is irreducible modulo $p$.

\section{REFERENCES}

[1] G. Azumaya, On maximally central algebras, Nagoya Math. J. 2 (1951), 119-150.

[2] F. De Meyer and E. Ingraham, Separable algebras over commutative rings, Lecture Notes in Math., vol. 181, Springer-Verlag, Berlin and New York, 1971.

[3] E. Dynkin, Semisimple subalgebras of semisimple Lie algebras, Mat. Sb. 30 (72) (1952), 349-462; English Transl., Amer. Math. Soc. Transl. 6 (1957), 111-244.

[4] _ Maximal subgroups of the classical groups, Trudy Moskov. Mat. Ob̌̌c. 1 (1952), 39-166; English transl., Amer. Math. Soc. Transl. 6 (1957), 245-378.

[5] O. T. O'Meara, Introduction to quadratic forms, Springer-Verlag, Heidelberg, 1963.

[6] M. Orzech and C. Small, The Brauer group of commutative rings, Marcel Dekker, New York, 1975.

[7] M. L. Racine, On maximal subalgebras, J. Algebra 30 (1974), 155-180.

[8] _ Maximal subalgebras of exceptional Jordan algebras, J. Algebra 46 (1977), 12-21.

[9] K. W. Roggenkamp and V. Huber-Dyson, Lattices over orders I, Lecture Notes in Math., vol. 115, Springer-Verlag, Berlin and New York, 1970.

Department of Mathematics, University of Ottawa, Ottawa, Ontario, Canada Kin 9B4 